\title{
A Plankton Allelopathic Model Described by a Delayed Quasilinear Parabolic System*
}

\author{
Canrong Tian ${ }^{a}$ and Peng $\mathbf{Z h u}^{b}$ \\ ${ }^{a}$ Yancheng Institute of Technology \\ Yancheng, 224003 Jiangsu, China \\ ${ }^{b}$ Yangzhou University \\ 225002 Yangzhou, China \\ E-mail(corresp.): unfoxeses@yahoo.com.cn \\ E-mail: zhupeng@ycit.edu.cn
}

Received July 14, 2011; revised April 28, 2012; published online September 1, 2012

\begin{abstract}
The quasilinear parabolic system has been applied to a variety of physical and engineering problems. However, most works lack effective techniques to deal with the asymptotic stability. This paper is concerned with the existence and stability of solutions for a plankton allelopathic model described by a quasilinear parabolic system, in which the diffusions are density-dependent. By the coupled upper and lower solutions and its associated monotone iterations, it is shown that under some parameter conditions the positive uniform equilibrium is asymptotically stable. Some biological interpretations for our results are given.
\end{abstract}

Keywords: parabolic reaction-diffusion equation, Volterra equation, quasi-linearization, global stability, existence.

AMS Subject Classification: 35B35; 92D50.

\section{Introduction}

We consider a coupled system of quasilinear parabolic equations in a bounded domain. The system of equations is given in the form

$$
\left\{\begin{array}{l}
\partial u_{1} / \partial t-\nabla \cdot\left(d_{1}\left(u_{1} / n_{1}\right)^{m} \nabla u_{1}\right)=u_{1}\left(a_{1}-b_{11} u_{1}-b_{12} u_{2}-e_{1} u_{1} u_{2}\right) \\
\quad(t>0, x \in \Omega), \\
\partial u_{2} / \partial t-\nabla \cdot\left(d_{2}\left(u_{2} / n_{2}\right)^{m} \nabla u_{2}\right)=u_{2}\left(a_{2}-b_{21} u_{1}-b_{22} u_{2}-e_{2}\left(u_{1}\right)_{\tau} u_{2}\right) \\
\quad(t>0, x \in \Omega), \\
\partial u_{1} / \partial \nu=\partial u_{2} / \partial \nu=0 \quad(t>0, x \in \partial \Omega), \\
u_{i}(t, x)=\psi_{i}(t, x) \quad(t \in[-\tau, 0], x \in \Omega), i=1,2,
\end{array}\right.
$$

* The work is partially supported by PRC grant NSFC 10801115 and also by NSFC 11101352. 
where $\Omega$ is a bounded domain in $\mathbb{R}^{n}$ with boundary $\partial \Omega, \partial / \partial \nu$ denotes the outward normal derivative on $\partial \Omega$. It is assumed that the boundary $\partial \Omega$ is of class $C^{1+\alpha} . \quad \psi_{i}(t, x) \in C^{\alpha / 2, \alpha}([-\tau, 0] \times \bar{\Omega})$ has a positive lower bound and satisfies the compatibility condition. $\left(u_{1}\right)_{\tau} \equiv u_{1}(t-\tau, x)$ is the discrete delay term. The density-dependent diffusion coefficient $D_{i}\left(u_{i}\right) \equiv d_{i}\left(u_{i} / n_{i}\right)^{m}(i=$ $1,2)$, in which $n_{i}>0, d_{i}>0$ and $m \geq 0$, has the property $D_{i}(0)=0$, which means the elliptic operators are degenerate. In the case $m=0,(1.1)$ is reduced to the semilinear parabolic system.

The system (1.1) is described by the plankton allelopathic competition model in aquatic ecology. $u_{1}, u_{2}$ stand for the population density (number of cells per liter) of two competing species; $a_{1}, a_{2}$ are the rates of cell proliferation per hour; $b_{11}, b_{22}$ are the rates of intra-specific competition of the first and the second species, respectively; $b_{12}, b_{21}$ are the rates of inter-specific competition of the first and the second species, respectively; $a_{i} / b_{i i}(i=1,2)$ are environmental carrying capacities (representing number of cell per liter). $e_{1}$ and $e_{2}$ are, respectively, the rates of toxic inhibition of the first species by the second and vice verse. The units of $a_{i}, b_{i j}$ and $e_{i}$ are per hour per cell and the unit of time is hours. The planktonic allelopathy model is investigated by ordinary differential equations $[2,9,12,11]$ and semilinear parabolic system [3, 23, 24, 25, 26]. Moreover, we incorporate the effect of disperse described by $D_{i}\left(u_{i}\right)=d_{i}\left(u_{i} / n_{i}\right)^{m}$ into the previous planktonic allelopathy model. Aikman and Hewitt have observed that the coefficients of diffusion are increasing when the densities of the populations are increasing in the experiment of dispersal patterns for grasshoppers [1]. Murray has first used the form $D(u)=d(u / n)^{m}$ to describe the density-dependent diffusion. The schematic solution obtained in [13] coincides with the experimental observation. Pao and Ruan have first introduced the density-dependent diffusion $D(u)=(n-1) u^{m-1}$ into Lotka-Volterra model [20]. They have investigated the existence, uniqueness and asymptotic behaviour of positive time-dependent solutions for the quasilinear parabolic system with quasimonotone nondecreasing reaction functions.

However, the requirement of the reaction functions in $[19,20,21]$ are quasimonotone nondecreasing. The condition is relaxed in this paper to mixed quasimonotone nondecreasing reaction functions, which leads to the difficult point that the ordered upper and lower solutions do not exist. To overcome it, we construct the coupled upper and lower solutions. In this paper, we aim to study the existence and asymptotic behaviour of (1.1) by the method of coupled upper and lower solutions.

The rest of this paper is organized as follows. In Section 2 we extend the monotone iteration method of [20] for quasimonotone nondecreasing reaction functions to delayed quasimonotone nondecreasing reaction functions. We show the existence and asymptotic stability of (1.1). In Section 3, we give the detail parameter conditions such that the positive uniform equilibrium is asymptotically stable. Section 4 is devoted to some discussions about the biological and mathematical senses. 


\section{Monotone Iteration Method}

In this section, we extend the monotone iteration method of [20] to delayed reaction functions. For the simplicity, throughout this paper, we denote

$$
\begin{gathered}
D=[0, \infty) \times \Omega, \quad \bar{D}=[0, \infty) \times \bar{\Omega}, \quad S=(0, \infty) \times \partial \Omega, \\
D_{0}^{(i)}=\left[-\tau_{i}, 0\right] \times \Omega, \quad Q^{(i)}=[-\tau, \infty) \times \Omega, \\
D_{0}=D_{0}^{(1)} \times D_{0}^{(2)}, \quad Q=Q^{(1)} \times Q^{(2)},
\end{gathered}
$$

and let $C^{m}(Q), C^{\alpha}(Q)$ be the respective space of $m$-times differentiable and Hölder continuous functions in $Q$, where $Q$ represents a domain. For vector functions with $N$-components we denote the above function space by $\mathcal{C}^{m}(Q)$ and $\mathcal{C}^{\alpha}(Q)$, respectively. We also denote

$$
\begin{aligned}
& f_{1}\left(u_{1}, u_{2}\right) \equiv u_{1}\left(a_{1}-b_{11} u_{1}-b_{12} u_{2}-e_{1} u_{1} u_{2}\right) \\
& f_{2}\left(u_{1}, u_{2},\left(u_{1}\right)_{\tau}\right) \equiv u_{2}\left(a_{2}-b_{21} u_{1}-b_{22} u_{2}-e_{2}\left(u_{1}\right)_{\tau} u_{2}\right) .
\end{aligned}
$$

Definition 1. A pair of function $\tilde{\mathbf{u}}=\left(\tilde{u}_{1}, \tilde{u}_{2}\right), \hat{\mathbf{u}}=\left(\hat{u}_{1}, \hat{u}_{2}\right) \in \mathcal{C}(\bar{Q}) \cap \mathcal{C}^{1,2}(Q)$ are called coupled upper and lower solutions of (1.1) if $\hat{\mathbf{u}}$ has a positive lower bound, and $\tilde{\mathbf{u}} \geq \hat{\mathbf{u}}$,

$$
\begin{aligned}
& \partial \tilde{u}_{1} / \partial t-\nabla \cdot\left(D_{1}\left(\tilde{u}_{1}\right) \nabla \tilde{u}_{1}\right) \geq f_{1}\left(\tilde{u}_{1}, \hat{u}_{2}\right) \quad \text { in } D, \\
& \partial \tilde{u}_{2} / \partial t-\nabla \cdot\left(D_{2}\left(\tilde{u}_{2}\right) \nabla \tilde{u}_{2}\right) \geq f_{2}\left(\hat{u}_{1}, \tilde{u}_{2},\left(\hat{u}_{1}\right)_{\tau}\right) \quad \text { in } D, \\
& \partial \hat{u}_{1} / \partial t-\nabla \cdot\left(D_{1}\left(\hat{u}_{1}\right) \nabla \hat{u}_{1}\right) \leq f_{1}\left(\hat{u}_{1}, \tilde{u}_{2}\right) \quad \text { in } D, \\
& \partial \hat{u}_{2} / \partial t-\nabla \cdot\left(D_{2}\left(\hat{u}_{2}\right) \nabla \hat{u}_{2}\right) \leq f_{2}\left(\tilde{u}_{1}, \hat{u}_{2},\left(\tilde{u}_{1}\right)_{\tau}\right) \quad \text { in } D, \\
& \partial \tilde{u}_{i} / \partial \nu \geq 0, \quad \partial \hat{u}_{i} / \partial \nu \leq 0, \quad i=1,2, \text { on } S, \\
& \tilde{u}_{i}(t, x) \geq \psi_{i}(t, x), \quad \hat{u}_{i}(t, x) \leq \psi_{i}(t, x), \quad i=1,2, \text { in } D_{0}^{(i)} .
\end{aligned}
$$

Define a modified function $\bar{D}_{i}\left(u_{i}\right)$ by

$$
\bar{D}_{i}\left(u_{i}\right)= \begin{cases}D_{i}\left(u_{i}\right)+\left(u_{i}-\tilde{u}_{i}\right) & \text { if } u_{i}>\tilde{u}_{i} \\ D_{i}\left(u_{i}\right) & \text { if } \hat{u}_{i} \leq u_{i} \leq \tilde{u}_{i} \\ D_{i}\left(u_{i}\right)+\left(\hat{u}_{i}-u_{i}\right) & \text { if } u_{i}<\hat{u}_{i}\end{cases}
$$

Then there exists $d_{0}>0$ such that $\bar{D}_{i}(u) \geq d_{0}$ for all $u \in \mathbb{R}$. Define

$$
w_{i}=I_{i}\left(u_{i}\right)=\int_{0}^{u_{i}} \bar{D}_{i}(s) d s \quad \text { for } u_{i} \geq 0, i=1,2,
$$

Derivativing (2.3), we have

$$
I_{i}^{\prime}\left(u_{i}\right)=d I_{i} / d u_{i}=\bar{D}_{i}\left(u_{i}\right)>0 .
$$

Then the inverse $u_{i} \equiv q_{i}\left(w_{i}\right)$ exists and is an increasing function of $w_{i}>0$. 
For a given pair of coupled upper and lower solutions $\tilde{\mathbf{u}}, \hat{\mathbf{u}}$ we set

$$
\begin{aligned}
& \Lambda_{i}=\left\{u_{i} \in C(\bar{Q}): \hat{u}_{i} \leq u_{i} \leq \tilde{u}_{i}\right\}, \quad \Lambda=\{\mathbf{u} \in \mathcal{C}(\bar{Q}): \hat{\mathbf{u}} \leq \mathbf{u} \leq \tilde{\mathbf{u}}\}, \\
& \Lambda \times \bar{\Lambda}=\{(\mathbf{u}, \mathbf{w}) \in \mathcal{C}(\bar{Q}) \times \mathcal{C}(\bar{Q}):(\hat{\mathbf{u}}, \hat{\mathbf{w}}) \leq(\mathbf{u}, \mathbf{w}) \leq(\tilde{\mathbf{u}}, \tilde{\mathbf{w}})\}
\end{aligned}
$$

where $\tilde{w}_{i}=I_{i}\left(\tilde{u}_{i}\right)$ and $\hat{w}_{i}=I\left(\hat{u}_{i}\right)$. There exist smooth nonnegative functions $\beta_{i} \equiv \beta_{i}(t, x)$ such that

$$
\beta_{i} \bar{D}_{i}\left(u_{i}\right)+\frac{\partial f_{i}}{\partial u_{i}}(\cdot, \mathbf{u}) \geq 0, \quad \text { for } \mathbf{u} \in \Lambda \text {. }
$$

In fact, it suffices to choose any $\beta_{i}(t, x)$ satisfying

$$
\beta_{i}(t, x) \geq \max \left\{-\left(\partial f_{i} / \partial u_{i}(t, x, \mathbf{u})\right) / \bar{D}_{i}\left(u_{i}\right): \mathbf{u} \in \Lambda\right\} .
$$

Define for each $i=1,2$,

$$
\begin{aligned}
& F_{1}\left(t, x, u_{1}, u_{2}\right)=\beta_{1}(t, x) I_{1}\left(u_{1}\right)+f_{1}\left(t, x, u_{1}, u_{2}\right), \\
& F_{2}\left(t, x, u_{1}, u_{2}\right)=\beta_{2}(t, x) I_{2}\left(u_{2}\right)+f_{2}\left(t, x, u_{1}, u_{2},\left(u_{1}\right)_{\tau}\right), \\
& L_{i} w_{i}=\nabla \cdot\left(\nabla w_{i}\right)-\beta_{i}(t, x) w_{i} .
\end{aligned}
$$

Since $(2.4),(2.5)$ and $I_{i}^{\prime}\left(u_{i}\right)=\bar{D}_{i}\left(u_{i}\right), F_{i}\left(\cdot, u_{i}, u_{j}\right)$ possess the monotone property

$$
F_{i}\left(\cdot, v_{i}, u_{j}\right) \leq F_{i}\left(\cdot, u_{i}, v_{j}\right) \quad \text { whenever } \quad \hat{\mathbf{u}} \leq \mathbf{v} \leq \mathbf{u} \leq \tilde{\mathbf{u}}
$$

We consider the system

$$
\begin{aligned}
& \left(\bar{D}_{i}\left(u_{i}\right)\right)^{-1} \partial w_{i} / \partial t-L_{i} w_{i}=F_{i}\left(t, x, u_{i}, u_{j}\right) \text { in } D \\
& \partial w_{i} / \partial \nu=0 \quad \text { on } S, \quad w_{i}(t, x)=\eta_{i}(t, x) \text { in } D_{0}^{(i)} \\
& u_{i}=q_{i}\left(w_{i}\right) \quad \text { for } i=1,2 \text { in } \bar{Q}^{(i)}
\end{aligned}
$$

where $\eta_{i}(t, x)=I_{i}\left(\psi_{i}(t, x)\right)$. Note that if $\bar{D}_{i}\left(u_{i}\right)=D_{i}\left(u_{i}\right)$, then $(2.7)$ is equivalent to (1.1).

By using $\underline{\mathbf{u}}^{(0)}=\hat{\mathbf{u}}$ and $\overline{\mathbf{u}}^{(0)}=\tilde{\mathbf{u}}$ as the initial iterations we can construct sequences $\left\{\underline{\mathbf{u}}^{(m)}, \underline{\mathbf{w}}^{(m)}\right\}$ and $\left\{\overline{\mathbf{u}}^{(m)}, \overline{\mathbf{w}}^{(m)}\right\}$ from the nonlinear iteration process

$$
\begin{aligned}
& \left(\bar{D}_{i}\left(\bar{u}_{i}^{(m)}\right)\right)^{-1} \partial \bar{w}_{i}^{(m)} / \partial t-L_{i} \bar{w}_{i}^{(m)}=F_{i}\left(t, x, \bar{u}_{i}^{(m-1)}, \underline{u}_{j}^{(m-1)}\right), \\
& \left(\bar{D}_{i}\left(\underline{u}_{i}^{(m)}\right)\right)^{-1} \partial \underline{w}_{i}^{(m)} / \partial t-L_{i} \underline{w}_{i}^{(m)}=F_{i}\left(t, x, \underline{u}_{i}^{(m-1)}, \bar{u}_{j}^{(m-1)}\right) \\
& \quad \text { for } i=1, j=2 ; \text { or } i=2, j=1 \text { in } D, \\
& \partial \bar{w}_{i}^{(m)} / \partial \nu=\partial \underline{w}_{i}^{(m)} / \partial \nu=0 \quad \text { for } i=1,2 \text { on } S, \\
& \bar{u}_{i}^{(m)}=q_{i}\left(\bar{w}_{i}^{(m)}\right), \quad \underline{u}_{i}^{(m)}=q_{i}\left(\underline{w}_{i}^{(m)}\right) \text { for } i=1,2 \text { in } \bar{Q}^{(i)}, \\
& \bar{w}_{i}^{(m)}(t, x)=\underline{w}_{i}^{(m)}(t, x)=\eta_{i}(t, x) \quad \text { for } i=1,2 \text { in } D_{0}^{(i)} .
\end{aligned}
$$

The sequences $\left\{\underline{\mathbf{u}}^{(m)}, \underline{\mathbf{w}}^{(m)}\right\}$ and $\left\{\overline{\mathbf{u}}^{(m)}, \overline{\mathbf{w}}^{(m)}\right\}$ are well defined by the existence theorem of [8]. The following lemma gives the monotone property of these sequences. 
Lemma 1. The sequences $\left\{\overline{\mathbf{u}}^{(m)}, \overline{\mathbf{w}}^{(m)}\right\},\left\{\underline{\mathbf{u}}^{(m)}, \underline{\mathbf{w}}^{(m)}\right\}$ governed by (2.8) possess the monotone property

$$
\begin{aligned}
(\hat{\mathbf{u}}, \hat{\mathbf{w}}) & \leq\left(\underline{\mathbf{u}}^{(\mathbf{m})}, \underline{\mathbf{w}}^{(\mathbf{m})}\right) \leq\left(\underline{\mathbf{u}}^{(\mathbf{m}+\mathbf{1})}, \underline{\mathbf{w}}^{(\mathbf{m}+\mathbf{1})}\right) \leq\left(\overline{\mathbf{u}}^{(\mathbf{m}+\mathbf{1})}, \overline{\mathbf{w}}^{(\mathbf{m}+\mathbf{1})}\right) \\
& \leq\left(\overline{\mathbf{u}}^{(\mathbf{m})}, \overline{\mathbf{w}}^{(\mathbf{m})}\right) \leq(\tilde{\mathbf{u}}, \tilde{\mathbf{w}}) \quad \text { for } m=1,2, \ldots
\end{aligned}
$$

Moreover, for each $m=1,2, \ldots, \overline{\mathbf{u}}^{(\mathbf{m})}$ and $\underline{\mathbf{u}}^{(\mathbf{m})}$ are coupled upper and lower solutions of (1.1).

Proof. Let $\underline{z}_{i}^{(1)}=\underline{w}_{i}^{(1)}-\underline{w}_{i}^{(0)}, i=1,2$. Then by (2.1) and (2.8), $\underline{z}_{i}^{(1)}$ satisfies

$$
\begin{aligned}
\left(\bar{D}_{i}\left(\underline{u}_{i}^{(1)}\right)\right)^{-1} \partial \underline{z}_{i}^{(1)} / \partial t-L_{i} \underline{z}_{i}^{(1)} & \\
= & F_{i}\left(\cdot, \underline{u}_{i}^{(0)}, \bar{u}_{j}^{(0)}\right)-\left[\left(\bar{D}_{i}\left(\underline{u}_{i}^{(1)}\right)\right)^{-1} \partial \underline{w}_{i}^{(0)} / \partial t-L_{i} \underline{w}_{i}^{(0)}\right] \\
= & F_{i}\left(\cdot, \underline{u}_{i}^{(0)}, \bar{u}_{j}^{(0)}\right)-\left[\left(\bar{D}_{i}\left(\underline{u}_{i}^{(0)}\right)\right)^{-1} \partial \underline{w}_{i}^{(0)} / \partial t-L_{i} \underline{w}_{i}^{(0)}\right] \\
& -\left[\left(\bar{D}_{i}\left(\underline{u}_{i}^{(1)}\right)\right)^{-1}-\left(\bar{D}_{i}\left(\underline{u}_{i}^{(0)}\right)\right)^{-1}\right] \partial \underline{w}_{i}^{(0)} / \partial t \\
\geq & -\left[\left(\bar{D}_{i}\left(\underline{u}_{i}^{(1)}\right)\right)^{-1}-\left(\bar{D}_{i}\left(\underline{u}_{i}^{(0)}\right)\right)^{-1}\right] \partial \underline{w}_{i}^{(0)} / \partial t .
\end{aligned}
$$

Since by the mean value theorem,

$$
\begin{aligned}
\left(\bar{D}_{i}\left(\underline{u}_{i}^{(1)}\right)\right)^{-1}-\left(\bar{D}_{i}\left(\underline{u}_{i}^{(0)}\right)\right)^{-1} & =-\left[\bar{D}_{i}^{\prime}\left(\xi^{(0)}\right) /\left(\bar{D}_{i}\left(\xi^{(0)}\right)\right)^{2}\right]\left(\underline{u}_{i}^{(1)}-\underline{u}_{i}^{(0)}\right) \\
& =-\left[\bar{D}_{i}^{\prime}\left(\xi^{(0)}\right) /\left(\bar{D}_{i}\left(\xi^{(0)}\right)\right)^{3}\right]\left(\underline{w}_{i}^{(1)}-\underline{w}_{i}^{(0)}\right),
\end{aligned}
$$

for some intermediate value $\xi^{(0)} \equiv \xi^{(0)}(t, x)$ between $\underline{u}_{i}^{(0)}$ and $\underline{u}_{i}^{(1)}$, we have

$$
\begin{aligned}
& \left(\bar{D}_{i}\left(\underline{u}_{i}^{(1)}\right)\right)^{-1} \partial \underline{z}_{i}^{(1)} / \partial t-L_{i} \underline{z}_{i}^{(1)}+\gamma^{(0)} \underline{z}_{i}^{(1)} \geq 0, \\
& \gamma^{(0)}=-\left[\bar{D}_{i}^{\prime}\left(\xi^{(0)}\right) /\left(\bar{D}_{i}\left(\xi^{(0)}\right)\right)^{3}\right] \partial \underline{w}_{i}^{(0)} / \partial t .
\end{aligned}
$$

And the boundary and initial inequalities satisfy

$$
\partial \underline{z}_{i}^{(1)} / \partial \nu=0 \quad \text { on } S, \quad \underline{z}_{i}^{(1)}(0, x)=\eta_{i}(0, x)-\eta_{i}(0, x)=0 \quad \text { in } \Omega .
$$

In view of the definition of $\bar{D}_{i}$ in $(2.2)$, the function $\bar{D}_{i}\left(\underline{u}_{i}^{(1)}\right) \gamma^{(0)}$ of $(2.10)$ is bounded. By Lemma 2.1 of $[20] \underline{z}_{i}^{(1)} \geq 0$ on $\bar{D}$. This gives $\underline{w}_{i}^{(1)} \geq \underline{w}_{i}^{(0)}$ and thus $\underline{u}_{i}^{(1)} \geq \underline{u}_{i}^{(0)}$. A similar argument yields $\bar{w}_{i}^{(1)} \leq \bar{w}_{i}^{(0)}$ and $\bar{u}_{i}^{(1)} \leq \bar{u}_{i}^{(0)}$.

Moreover, letting $z_{i}^{(1)}=\bar{w}_{i}^{(1)}-\underline{w}_{i}^{(1)}$, by (2.8), and after the similar above argument

$$
\begin{aligned}
& \left(\bar{D}_{i}\left(\bar{u}_{i}^{(1)}\right)\right)^{-1} \partial z_{i}^{(1)} / \partial t-L_{i} z_{i}^{(1)}+\gamma_{i}^{(0)} z_{i}^{(1)} \\
& \quad=F_{i}\left(\cdot, \bar{u}_{i}^{(0)}, \underline{u}_{j}^{(0)}\right)-F_{i}\left(\cdot, \underline{u}_{i}^{(0)}, \bar{u}_{j}^{(0)}\right) \geq 0 \quad \text { in } D \\
& \partial z_{i}^{(1)} / \partial \nu=0 \quad \text { on } S, \quad z_{i}^{(1)}(0, x)=\eta_{i}(0, x)-\eta_{i}(0, x)=0 \quad \text { in } \Omega,
\end{aligned}
$$

where $\gamma_{i}^{(0)}=-\left[\bar{D}_{i}^{\prime}\left(\xi_{i}^{(0)}\right) /\left(\bar{D}_{i}\left(\xi_{i}^{(0)}\right)\right)^{3}\right] \partial \underline{w}_{i}^{(0)} / \partial t$, for some intermediate value $\xi_{i}^{(0)} \equiv \xi_{i}^{(0)}(t, x)$ between $\underline{u}_{i}^{(0)}$ and $\bar{u}_{i}^{(0)}$. It follows again from Lemma 2.1 of [20] that $\overline{\mathbf{w}}^{(1)} \geq \underline{\mathbf{w}}^{(1)}$ and thus $\overline{\mathbf{u}}^{(1)} \geq \underline{\mathbf{u}}^{(1)}$. The above conclusions show that

$$
\left(\underline{\mathbf{u}}^{(0)}, \underline{\mathbf{w}}^{(0)}\right) \leq\left(\underline{\mathbf{u}}^{(1)}, \underline{\mathbf{w}}^{(1)}\right) \leq\left(\overline{\mathbf{u}}^{(1)}, \overline{\mathbf{w}}^{(1)}\right) \leq\left(\overline{\mathbf{u}}^{(0)}, \overline{\mathbf{w}}^{(0)}\right) .
$$


Now we show that $\overline{\mathbf{u}}^{(1)}$ and $\underline{\mathbf{u}}^{(1)}$ are coupled upper and lower solutions of (1.1). Since $(2.2)$ and $(2.12), \bar{D}_{i}\left(\bar{u}_{i}^{(1)}\right)=D_{i}\left(\bar{u}_{i}^{(1)}\right)$ for $i=1,2$. It suffices to show $\overline{\mathbf{u}}^{(1)}$ and $\underline{\mathbf{u}}^{(1)}$ satisfy (2.1). Since (2.6), (2.8) and (2.11), we have

$$
\begin{aligned}
& \left(D_{i}\left(\bar{u}_{i}^{(1)}\right)\right)^{-1} \partial \bar{w}_{i}^{(1)} / \partial t-L_{i} \bar{w}_{i}^{(1)}=F_{i}\left(\cdot, \bar{u}_{i}^{(0)}, \underline{u}_{j}^{(0)}\right) \geq F_{i}\left(\cdot, \bar{u}_{i}^{(1)}, \underline{u}_{j}^{(1)}\right), \\
& \left(D_{i}\left(\underline{u}_{i}^{(1)}\right)\right)^{-1} \partial \underline{w}_{i}^{(1)} / \partial t-L_{i} \underline{w}_{i}^{(1)}=F_{i}\left(\cdot, \cdot, \underline{u}_{i}^{(0)}, \bar{u}_{j}^{(0)}\right) \leq F_{i}\left(\cdot, \underline{u}_{i}^{(1)}, \bar{u}_{j}^{(1)}\right), \\
& \partial \bar{u}_{i}^{(1)} / \partial \nu=\partial \underline{u}_{i}^{(1)} / \partial \nu=0, \quad \bar{u}_{i}^{(1)}(0, x)=\eta_{i}(0, x), \quad \underline{u}_{i}^{(1)}(0, x)=\eta_{i}(0, x) .
\end{aligned}
$$

Next we use an induction method. By choosing $\overline{\mathbf{u}}^{(1)}$ and $\underline{\mathbf{u}}^{(1)}$ as the coupled upper and lower solutions $\tilde{\mathbf{u}}$ and $\hat{\mathbf{u}}$, after the similar above argument, we have

$$
\left(\underline{\mathbf{u}}^{(1)}, \underline{\mathbf{w}}^{(1)}\right) \leq\left(\underline{\mathbf{u}}^{(2)}, \underline{\mathbf{w}}^{(2)}\right) \leq\left(\overline{\mathbf{u}}^{(2)}, \overline{\mathbf{w}}^{(2)}\right) \leq\left(\overline{\mathbf{u}}^{(1)}, \overline{\mathbf{w}}^{(1)}\right),
$$

$\overline{\mathbf{u}}^{(2)}$ and $\underline{\mathbf{u}}^{(2)}$ are coupled upper and lower solutions of (1.1). The conclusion of the lemma follows from the induction principle.

In view of Lemma 1 , the pointwise limits

$$
\lim _{m \rightarrow \infty}\left(\overline{\mathbf{u}}^{(\mathbf{m})}, \overline{\mathbf{w}}^{(\mathbf{m})}\right)=(\overline{\mathbf{u}}, \overline{\mathbf{w}}), \quad \lim _{m \rightarrow \infty}\left(\underline{\mathbf{u}}^{(\mathbf{m})}, \underline{\mathbf{w}}^{(\mathbf{m})}\right)=(\underline{\mathbf{u}}, \underline{\mathbf{w}})
$$

exist. Since $(2.2)$ and $(2.9), \bar{D}_{i}\left(\bar{u}_{i}^{(m)}\right)=D_{i}\left(\bar{u}_{i}^{(m)}\right)$ for $i=1,2$. Then by letting $m \rightarrow \infty$ in (2.8), we obtain the following system

$$
\begin{aligned}
& \partial \bar{u}_{1} / \partial t-\nabla \cdot\left(D_{1}\left(\bar{u}_{1}\right) \nabla \bar{u}_{1}\right)=\bar{u}_{1}\left(a_{1}-b_{11} \bar{u}_{1}-b_{12} \underline{u}_{2}-e_{1} \bar{u}_{1} \underline{u}_{2}\right), \\
& \partial \bar{u}_{2} / \partial t-\nabla \cdot\left(D_{2}\left(\bar{u}_{2}\right) \nabla \bar{u}_{2}\right)=\bar{u}_{2}\left(a_{2}-b_{21} \underline{u}_{1}-b_{22} \bar{u}_{2}-e_{2}\left(\underline{u}_{1}\right)_{\tau} \bar{u}_{2}\right), \\
& \partial \underline{u}_{1} / \partial t-\nabla \cdot\left(D_{1}\left(\underline{u}_{1}\right) \nabla \underline{u}_{1}\right)=\underline{u}_{1}\left(a_{1}-b_{11} \underline{u}_{1}-b_{12} \bar{u}_{2}-e_{1} \underline{u}_{1} \bar{u}_{2}\right), \\
& \partial \underline{u}_{2} / \partial t-\nabla \cdot\left(D_{2}\left(\underline{u}_{2}\right) \nabla \underline{u}_{2}\right)=\underline{u}_{2}\left(a_{2}-b_{21} \bar{u}_{1}-b_{22} \underline{u}_{2}-e_{2}\left(\bar{u}_{1}\right)_{\tau} \underline{u}_{2}\right) \quad \text { in } Q, \\
& \partial \bar{u}_{i} / \partial \nu=\partial \underline{u}_{i} / \partial \nu=0 \quad \text { for } i=1,2, \text { on } S, \\
& \bar{u}_{i}(t, x)=\underline{u}_{i}(t, x)=\psi_{i}(t, x) \quad \text { for } i=1,2, \text { in } D_{0}^{(i)} .
\end{aligned}
$$

Next we show that the $(\overline{\mathbf{u}}, \underline{\mathbf{u}})$ are the solutions of (2.13) using the standard regularity argument.

Lemma 2. $(\overline{\mathbf{u}}(t, x), \underline{\mathbf{u}}(t, x))$ is the solution of $(2.13)$.

Proof. Since $(2.8), \bar{u}_{i}^{(m)}(i=1, \ldots, N)$ is a solution of the scalar quasilinear system

$$
\begin{aligned}
& \partial u / \partial t-\nabla \cdot\left(a_{i} D_{i}(u) \nabla u\right)+\mathbf{b}_{\mathbf{i}} \cdot\left(D_{i}(u) \nabla u\right)+\beta_{i} I_{i}(u) \\
& \quad=F_{i}\left(\cdot, \bar{u}_{i}^{(m-1)}, \bar{u}_{i}^{(m-1)}, \underline{u}_{j}^{(m-1)}\right) \quad \text { in } D \\
& \partial u / \partial \nu=0 \quad \text { on } S, \quad u(t, x)=\psi_{i}(t, x) \quad \text { in } D_{0}^{(i)}
\end{aligned}
$$

by hypothesis $\left(H_{1}\right)$ and Theorem 7.4 of Chapter $\mathrm{V}$ in [8], there is a constant $\alpha>0$ such that $\bar{u}_{i}^{(m)} \in C^{1+\alpha / 2,2+\alpha}(\bar{Q})$ for all $m=1,2, \ldots$ Furthermore, since 
the sequence $\bar{u}_{i}^{(m)}$ is uniformly bounded in $C(\bar{Q})$, it follows from Theorem 7.2 of Chapter $\mathrm{V}$ in [8] that there exist positive constants $M$ and $\delta$, independent of $m$, such that

$$
\left|\bar{u}_{i}^{(m)}\right|_{C^{1+\alpha / 2,2+\alpha}(\bar{Q})} \leq M, \quad\left|\nabla \bar{u}_{i}^{(m)}\right|_{C(\bar{Q})} \leq M .
$$

We now show that the limit $\bar{u}_{i}$ of $\bar{u}_{i}^{(m)}$ satisfies the first equation of (2.13) in $Q$. For each $\bar{u}_{i}^{(m)}$, we let the operator $\mathcal{L}^{(m)}$ and the function $\mathcal{F}^{(m)}$ be defined by

$$
\begin{aligned}
\mathcal{L}^{(m)} & =\partial u / \partial t-\nabla \cdot\left(a_{i} D_{i}(u) \nabla u\right)+\mathbf{b}_{\mathbf{i}} \cdot\left(D_{i}(u) \nabla u\right) \\
& \equiv \partial u / \partial t-a_{i} D_{i}(u) \nabla^{2} u+\left[D_{i}(u) \nabla a_{i}+a_{i} D_{i}^{\prime}(u) \nabla u+D_{i}(u) \mathbf{b}_{\mathbf{i}}\right] \cdot \nabla u \\
\mathcal{F}^{(m)} & =F_{i}\left(\cdot, \bar{u}_{i}^{(m-1)}, \bar{u}_{i}^{(m-1)}, \underline{u}_{j}^{(m-1)}\right)-\beta_{i} I_{i}(u(t, x)) .
\end{aligned}
$$

In view of $(2.11), \bar{u}_{i}^{(m)}$ satisfies the linear equation $\mathcal{L}^{(m)} u=\mathcal{F}^{m}$ in $Q$. Let $Q^{\prime}$ be an arbitrary subdomain of $Q$ whose distance from $Q$ has a positive lower bound. Then by (2.15), all the conditions in Theorem 15 of [4] are satisfied in $Q^{\prime}$. Hence there is a subsequence $\bar{u}^{\left(m^{\prime}\right)}$ such that $\nabla \bar{u}^{\left(m^{\prime}\right)}, \nabla^{2} \bar{u}^{\left(m^{\prime}\right)}$, and $\partial \bar{u}^{\left(m^{\prime}\right)} / \partial t$ are all uniformly convergent in $\bar{Q}_{T}^{\prime}$, and the coefficients of $\mathcal{L}^{(m)}$ converge to the corresponding limits. This proves that the limit $\bar{u}_{i}$ is in $C^{1,2}\left(\bar{Q}^{\prime}\right)$ and satisfies the equation

$$
\frac{\partial u}{\partial t}-\nabla \cdot\left(a_{i} D_{i}(u) \nabla u\right)+\mathbf{b}_{\mathbf{i}} \cdot\left(D_{i}(u) \nabla u\right)=f_{i}\left(\cdot, \bar{u}_{i}^{(m-1)}, \bar{u}_{i}^{(m-1)}, \underline{u}_{j}^{(m-1)}\right) \text { in } Q^{\prime} .
$$

Since $Q^{\prime}$ is arbitrary, $\bar{u}_{i}$ satisfies the first equation of (2.13) in the whole domain $Q$. Similarly, $\underline{u}_{i}$ also satisfies the second equation of (2.13). Thus the proof is completed.

We call $\overline{\mathbf{u}}, \underline{\mathbf{u}}$ quasisolutions of (1.1). Moreover, by observing the first and fourth equation of (2.13), we conclude that $\left(\bar{u}_{1}, \underline{u}_{2}\right)$ is the solution of (1.1). Similarly, $\left(\underline{u}_{1}, \bar{u}_{2}\right)$ is also the solution of (1.1). It thus leads to the existence theorem from Lemma 2.

Theorem 1. Let $\tilde{\mathbf{u}}, \hat{\mathbf{u}}$ be a pair of coupled upper and lower solutions of (1.1). Assume that $\hat{\mathbf{u}} \geq \delta>0$, then

(i) $\left(\bar{u}_{1}, \underline{u}_{2}\right)$ and $\left(\underline{u}_{1}, \bar{u}_{2}\right)$ are the solutions of (1.1). Moreover for all $m \geq 1$

$$
\hat{\mathbf{u}} \leq \underline{\mathbf{u}}^{(\mathbf{m})} \leq \underline{\mathbf{u}}^{(\mathbf{m}+\mathbf{1})} \leq \underline{\mathbf{u}} \leq \overline{\mathbf{u}} \leq \overline{\mathbf{u}}^{(\mathbf{m}+\mathbf{1})} \leq \overline{\mathbf{u}}^{(\mathbf{m})} \leq \tilde{\mathbf{u}} \text { in } \bar{D} .
$$

(ii) If $\underline{\mathbf{u}}=\overline{\mathbf{u}}\left(\equiv \mathbf{u}^{*}\right)$, then $\mathbf{u}^{*}$ is the unique solution in $\Lambda$.

Next we discuss the asymptotic behaviour of the problem (1.1) by using the method as in $[15,16,17,18]$. Assume that the coupled upper and lower solutions are constant vectors $\tilde{\mathbf{c}} \equiv\left(\tilde{c}_{1}, \tilde{c}_{2}\right), \hat{\mathbf{c}} \equiv\left(\hat{c}_{1}, \hat{c}_{2}\right)$. By using $\underline{\mathbf{c}}^{(0)}=\hat{\mathbf{c}}$ and $\overline{\mathbf{c}}^{(0)}=\tilde{\mathbf{c}}$ as the initial iteration, after the nonlinear iteration process $(2.8)$, we can construct constant sequences $\left\{\underline{\mathbf{c}}^{(m)}, \underline{\mathbf{c}}^{(m)}\right\}$ and $\left\{\overline{\mathbf{c}}^{(m)}, \overline{\mathbf{c}}^{(m)}\right\}$. We set the pointwise limits

$$
\lim _{m \rightarrow \infty}\left(\bar{c}_{1}^{(m)}, \bar{c}_{2}^{(m)}\right)=\left(\bar{c}_{1}, \bar{c}_{2}\right), \quad \lim _{m \rightarrow \infty}\left(\underline{c}_{1}^{(m)}, \underline{c}_{2}^{(m)}\right)=\left(\underline{c}_{1}, \underline{c}_{2}\right) .
$$


Such as in $(2.16),\left(\bar{c}_{1}, \bar{c}_{2}\right)$ and $\left(\underline{c}_{1}, \underline{c}_{2}\right)$ satisfy the following relation

$$
\begin{aligned}
& \bar{c}_{1}\left(a_{1}-b_{11} \bar{c}_{1}-b_{12} \underline{c}_{2}-e_{1} \bar{c}_{1} \underline{c}_{2}\right)=0=\underline{c}_{1}\left(a_{1}-b_{11} \underline{c}_{1}-b_{12} \bar{c}_{2}-e_{1} \underline{c}_{1} \bar{c}_{2}\right), \\
& \bar{c}_{2}\left(a_{2}-b_{21} \underline{c}_{1}-b_{22} \bar{c}_{2}-e_{2} \underline{c}_{1} \bar{c}_{2}\right)=0=\underline{c}_{2}\left(a_{2}-b_{21} \bar{c}_{1}-b_{22} \underline{c}_{2}-e_{2} \bar{c}_{1} \underline{c}_{2}\right) .
\end{aligned}
$$

After the similar argument as in the proof of Lemma 2, we have the following asymptotic property.

Theorem 2. Let $\tilde{\mathbf{c}}$, $\hat{\mathbf{c}}$ be a pair of coupled constant upper and lower solutions of (1.1). Assume that $\hat{\mathbf{c}} \geq \delta>0$, then the quasisolutions $\left(\bar{c}_{1}, \bar{c}_{2}\right)$ and $\left(\underline{c}_{1}, \underline{c}_{2}\right)$ satisfy (2.17) and

$\hat{\mathbf{c}} \leq \underline{\mathbf{c}}^{(\mathbf{m})} \leq \underline{\mathbf{c}}^{(\mathbf{m}+\mathbf{1})} \leq \underline{\mathbf{c}} \leq \overline{\mathbf{c}} \leq \overline{\mathbf{c}}^{(\mathbf{m}+\mathbf{1})} \leq \overline{\mathbf{c}}^{(\mathbf{m})} \leq \tilde{\mathbf{c}} \quad$ in $\bar{Q}, m=1,2, \ldots$

If $\left(\bar{c}_{1}, \bar{c}_{2}\right)=\left(\underline{c}_{1}, \underline{c}_{2}\right) \equiv\left(c_{1}^{*}, c_{2}^{*}\right)$, then for any $\hat{c}_{i} \leq \psi_{i}(t, x) \leq \tilde{c}_{i}$ in $D_{i}$, the unique solution $\left(u_{1}(t, x), u_{2}(t, x)\right)$ possesses the convergence property is the unique solution

$$
\lim _{t \rightarrow \infty}\left(u_{1}(t, x), u_{2}(t, x)\right)=\left(c_{1}^{*}, c_{2}^{*}\right) \quad(x \in \bar{\Omega}) .
$$

\section{Existence and Asymptotic Behavior of Solutions}

First we show the existence by seeking the coupled upper and lower solutions of (1.1). It is easy to verify that if $\left(\tilde{u}_{1}, \tilde{u}_{2}\right)$ and $\left(\hat{u}_{1}, \hat{u}_{2}\right)$ satisfy $\left(\tilde{u}_{1}, \tilde{u}_{2}\right) \geq\left(\hat{u}_{1}, \hat{u}_{2}\right)$ and the following inequalities

$$
\begin{aligned}
& \partial \tilde{u}_{1} / \partial t-\nabla \cdot\left(D_{1}\left(\tilde{u}_{1}\right) \nabla \tilde{u}_{1}\right) \geq \tilde{u}_{1}\left(a_{1}-b_{11} \tilde{u}_{1}-b_{12} \hat{u}_{2}-e_{1} \tilde{u}_{1} \hat{u}_{2}\right), \\
& \partial \tilde{u}_{2} / \partial t-\nabla \cdot\left(D_{2}\left(\tilde{u}_{2}\right) \nabla \tilde{u}_{2}\right) \geq \tilde{u}_{2}\left(a_{2}-b_{21} \hat{u}_{1}-b_{22} \tilde{u}_{2}-e_{2}\left(\hat{u}_{1}\right)_{\tau} \tilde{u}_{2}\right), \\
& \partial \hat{u}_{1} / \partial t-\nabla \cdot\left(D_{1}\left(\hat{u}_{1}\right) \nabla \hat{u}_{1}\right) \leq \hat{u}_{1}\left(a_{1}-b_{11} \hat{u}_{1}-b_{12} \tilde{u}_{2}-e_{1} \hat{u}_{1} \tilde{u}_{2}\right), \\
& \partial \hat{u}_{2} / \partial t-\nabla \cdot\left(D_{2}\left(\hat{u}_{2}\right) \nabla \hat{u}_{2}\right) \leq \hat{u}_{2}\left(a_{2}-b_{21} \tilde{u}_{1}-b_{22} \hat{u}_{2}-e_{2}\left(\tilde{u}_{1}\right)_{\tau} \hat{u}_{2}\right) \quad \text { in } D, \\
& \partial \hat{u}_{i} / \partial \nu \leq 0 \leq \partial \tilde{u}_{i} / \partial \nu \quad \text { for } i=1,2 \text {, on } S, \\
& \hat{u}_{i}(t, x) \leq \psi_{i}(t, x) \leq \tilde{u}_{i}(t, x) \text { for } i=1,2, \text { in } D_{i} .
\end{aligned}
$$

then the pair $\left(\tilde{u}_{1}, \tilde{u}_{2}\right),\left(\hat{u}_{1}, \hat{u}_{2}\right)$ are coupled upper and lower solutions of (1.1). To guarantee (3.1), we seek such a constant pair in the form $\left(\tilde{c}_{1}, \tilde{c}_{2}\right)=\left(M_{1}, M_{2}\right)$, $\left(\hat{c}_{1}, \hat{c}_{2}\right)=\left(\delta_{1}, \delta_{2}\right)$, where for each $i=1,2, M_{i}$ are positive constants and $\delta_{i}$ are some sufficiently small constants. Thus (3.1) is satisfied if

$$
\begin{gathered}
\tilde{c}_{1}\left(a_{1}-b_{11} \tilde{c}_{1}-b_{12} \hat{c}_{2}-e_{1} \tilde{c}_{1} \hat{c}_{2}\right) \leq 0 \leq \hat{c}_{1}\left(a_{1}-b_{11} \hat{c}_{1}-b_{12} \tilde{c}_{2}-e_{1} \hat{c}_{1} \tilde{c}_{2}\right), \\
\tilde{c}_{2}\left(a_{2}-b_{21} \hat{c}_{1}-b_{22} \tilde{c}_{2}-e_{2} \hat{c}_{1} \tilde{c}_{2}\right) \leq 0 \leq \hat{c}_{2}\left(a_{2}-b_{21} \tilde{c}_{1}-b_{22} \hat{c}_{2}-e_{2} \tilde{c}_{1} \hat{c}_{2}\right), \\
\psi_{i}(t, x) \leq \tilde{c}_{i} \text { for } i=1,2 \text {, in } D_{0}^{(i)} .
\end{gathered}
$$

If we set $M_{1}=a_{1} / b_{11}<a_{2} / b_{21}, M_{2}=a_{2} / b_{22}<a_{1} / b_{12}$, then (3.2) is satisfied. Therefore we conclude the existence results.

Theorem 3. Suppose there exists a positive constant $\delta$ such that the initial functions $\delta \leq \psi_{i}(x, t)<a_{i} / b_{i i}(i=1,2)$ in $D_{0}^{(i)}$. If

$$
b_{12} / b_{22}<a_{1} / a_{2}<b_{11} / b_{21},
$$

then the system (1.1) admits at least one positive solution. 
In order to investigate the asymptotic behaviour of the coupled system (1.1), we consider the scalar logistic parabolic equation

$$
\left\{\begin{array}{l}
\partial u_{1} / \partial t-\nabla \cdot\left(d_{1}\left(u_{1} / n_{1}\right)^{m} \nabla u_{1}\right)=u_{1}\left(a_{1}-b_{11} u_{1}\right) \quad(t>0, x \in \Omega), \\
\partial u_{1} / \partial \nu=0 \quad(t>0, x \in \partial \Omega), \\
u_{1}(t, x)=\psi_{1}(t, x) \quad(t \in[-\tau, 0], x \in \Omega) .
\end{array}\right.
$$

Lemma 3. There exists the unique solution $u_{1}(x, t)$ of (3.4), which satisfies

$$
\lim _{t \rightarrow \infty} u_{1}(x, t)=a_{1} / b_{11}
$$

Proof. It is obvious that

$$
\tilde{c}_{1}=\max \left\{a_{1} / b_{11}, \max _{(x, t) \in D_{1}} \psi_{1}(t, x)\right\}, \quad \hat{c}_{1}=\min _{(x, t) \in D_{1}} \psi_{1}(t, x)
$$

are upper and lower solutions of (3.4). Thus the existence of $u_{1}(x, t)$ is ensured by Theorem 1. Applying Theorem 2, (2.17) is reduced to

$$
\bar{c}_{1}\left(a_{1}-b_{11} \bar{c}_{1}\right)=0=\underline{c}_{1}\left(a_{1}-b_{11} \underline{c}_{1}\right) .
$$

Since (2.16), $0<\underline{c}_{1}<\bar{c}_{1}$ holds. Thus it follows from (3.5) that $\underline{c}_{1}=\bar{c}_{1}=$ $a_{1} / b_{11}$, which proves the theorem by using Theorem 2 . lemma.

We now give the global attractivity of the system (1.1) in the following

Lemma 4. For any given initial function $\psi_{i}(x, t) \geq \delta$ in $D_{0}^{(i)}$ for $i=1,2$, if (3.3) holds, then the solution $\left(u_{1}(x, t), u_{2}(x, t)\right)$ of the system (1.1) satisfies

$$
\lim _{t \rightarrow \infty} u_{1}(x, t) \leq \frac{a_{1}}{b_{11}}, \quad \lim _{t \rightarrow \infty} u_{2}(x, t) \leq \frac{a_{2}}{b_{22}} \quad \text { in } Q
$$

Proof. We essentially use the positive lemma of Lemma 2.1 in [20]. Considering the scalar quasilinear parabolic problem as the following

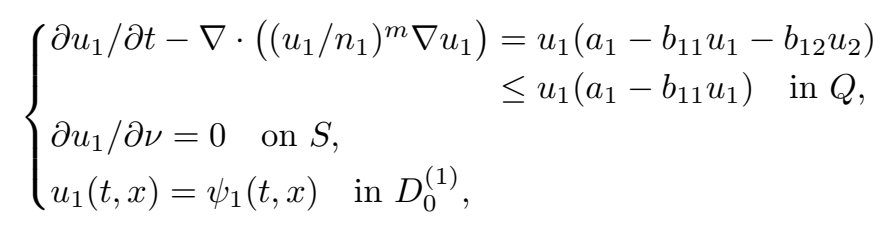

in view of the positive lemma that $u_{1}(t, x) \leq U_{1}(t, x)$, where $U_{1}(t, x)$ is the unique solution of (3.4). Letting $t \rightarrow \infty, \lim _{t \rightarrow \infty} u_{1}(x, t) \leq \lim _{t \rightarrow \infty} U_{1}(x, t) \leq$ $a_{1} / b_{11}$. Similarly, $\lim _{t \rightarrow \infty} u_{2}(x, t) \leq a_{2} / b_{22}$. Thus the proof is completed.

From Lemma 4, for any initial functions, the solution of (1.1) will go into the attractor $\left[0, a_{1} / b_{11}\right] \times\left[0, a_{2} / b_{22}\right]$. Therefore in this case Theorem 3 is valid.

Corollary 1. If (3.3) holds, then the system (1.1) admits at least one positive solution. Moreover, (3.6) holds. 
Now we study the global stability of the uniform equilibrium for (1.1). After some algebra calculations similar as in the argument in [12], we get the sufficient conditions such that system (1.1) has the positive uniform equilibrium, moreover the positive uniform equilibrium is locally stable.

Lemma 5. If

$$
b_{12} / b_{22}<a_{1} / a_{2}<b_{11} / b_{21}, \quad b_{12} / b_{22}<e_{1} / e_{2}<b_{11} / b_{21},
$$

then (1.1) admits the unique positive uniform equilibrium $E^{*}:\left(N_{1}^{*}, N_{2}^{*}\right)$, where $N_{1}^{*}, N_{2}^{*}$ are the positive constants depending on the coefficients $a_{i}, b_{i j}, e_{i}$.

Lemma 6. If (3.7) holds, then the positive uniform equilibrium $E^{*}:\left(N_{1}^{*}, N_{2}^{*}\right)$ of (1.1) is locally stable.

Proof. First, for sake of simplicity, we denote

$$
\mathbf{f}(\mathbf{u})=\left(\begin{array}{c}
f_{1}\left(u_{1}, u_{2}\right) \\
f_{2}\left(u_{1}, u_{2},\left(u_{1}\right)_{\tau}\right)
\end{array}\right) .
$$

The linearization of (1.1) around the state $E^{*}$ gives

$$
\partial \mathbf{u} / \partial t-D \Delta \mathbf{u}^{m+1}=\mathbf{f}_{\mathbf{u}}\left(E^{*}\right) \mathbf{u}
$$

where $D=\operatorname{diag}\left(d_{1} /(m+1) n_{1}^{m}, d_{2} /(m+1) n_{2}^{m}\right)$, and

$$
\mathbf{f}_{\mathbf{u}}\left(E^{*}\right)=\left(\begin{array}{cc}
-N_{1}^{*}\left(b_{11}+e_{1} N_{2}^{*}\right) & -N_{1}^{*}\left(b_{12}+e_{1} N_{1}^{*}\right) \\
-N_{2}^{*}\left(b_{21}+e_{2} N_{2}^{*} e^{-\lambda \tau}\right) & -N_{2}^{*}\left(b_{22}+e_{2} N_{1}^{*}\right)
\end{array}\right) \triangleq\left(\begin{array}{ll}
\mathbf{f}_{\mathbf{u} 11} & \mathbf{f}_{\mathbf{u} 12} \\
\mathbf{f}_{\mathbf{u} 21} & \mathbf{f}_{\mathbf{u} 22}
\end{array}\right) .
$$

Setting $0=\mu_{1}<\mu_{2}<\cdots \rightarrow \infty$ be the eigenvalues of $-\Delta \mathbf{u}^{m+1}$ on $\Omega$ under no-flux boundary conditions, direct calculation shows that the characteristic polynomial of (3.8) is given by

$$
\psi_{i}(\lambda)=\lambda^{2}-B_{i} \lambda+C_{i}
$$

where

$$
\begin{aligned}
B_{i}= & -N_{1}^{*}\left(b_{11}+e_{1} N_{2}^{*}\right)-N_{2}^{*}\left(b_{22}+e_{2} N_{1}^{*}\right)-d_{1} \mu_{i}-d_{2} \mu_{i}, \\
C_{i}= & \left(N_{1}^{*}\left(b_{11}+e_{1} N_{2}^{*}\right)+d_{1} \mu_{i}\right)\left(N_{2}^{*}\left(b_{22}+e_{2} N_{1}^{*}\right)+d_{2} \mu_{i}\right) \\
& -N_{1}^{*} N_{2}^{*}\left(b_{12}+e_{1} N_{1}^{*}\right)\left(b_{21}+e_{2} N_{2}^{*} e^{-\lambda \tau}\right) .
\end{aligned}
$$

Recalling condition (3.7), it is easy to verify that $B_{i}$ and $C_{i}$ are negative. Thus, for each $i \geq 1$, the two roots $\lambda_{i, 1}, \lambda_{i, 2}$ of $\psi_{i}(\lambda)=0$ all have negative real parts, and this concludes the proof.

Based on the local stability of the equilibrium of (1.1), we can have the global stability of the equilibrium by using of Theorem 2 .

Theorem 4. Suppose there exists a positive constant $\delta$ such that the initial functions $\delta \leq \psi_{i}(x, t)(i=1,2)$ in $D_{0}^{(i)}$. If (3.7) holds, then the positive $E^{*}:\left(N_{1}^{*}, N_{2}^{*}\right)$ is asymptotically stable. 
Proof. In view of Lemma 4, we have the following estimates

$$
\lim _{t \rightarrow \infty} u_{1}(x, t) \leq a_{1} / b_{11}, \quad \lim _{t \rightarrow \infty} u_{2}(x, t) \leq a_{2} / b_{22} .
$$

For any given $\varepsilon_{1}, \varepsilon_{2}>0$, there exists $t_{0}>0$ such that $u_{1}(x, t) \leq a_{1} / b_{11}+\varepsilon_{1}$, $u_{2}(x, t) \leq a_{2} / b_{22}+\varepsilon_{2}$. As the asymptotic behaviour is concerned, it suffices to consider the case $t \geq t_{0}$. Hence the globally asymptotic behaviour of system (1.1) for any initial function is equivalent to $0<\eta_{i}(x, t) \leq a_{i} / b_{i i}+\varepsilon_{i}(i=1,2)$. In order to apply the conclusions of Theorem 2 , we seek the coupled upper and lower solutions $\tilde{\mathbf{c}}$ and $\hat{\mathbf{c}}$.

We set $\tilde{c}_{1}=a_{1} / b_{11}+\varepsilon_{1}, \tilde{c}_{2}=a_{2} / b_{22}+\varepsilon_{2}, \hat{c}_{1}=\delta_{1}, \hat{c}_{2}=\delta_{2}$, where

$$
\begin{aligned}
& \varepsilon_{1}=a_{2} b_{11}-a_{1} b_{21} / 2 b_{11} b_{21}, \quad \varepsilon_{2}=a_{1} b_{22}-a_{2} b_{12} / 2 b_{12} b_{22}, \\
& \delta_{1} \leq b_{12}\left(a_{1} b_{22}-a_{2} b_{12}\right) /\left[2 b_{11} b_{12} b_{22}+e_{1}\left(a_{1} b_{22}+a_{2} b_{12}\right)\right], \\
& \delta_{2} \leq b_{21}\left(a_{2} b_{11}-a_{1} b_{21}\right) /\left[2 b_{11} b_{21} b_{22}+e_{2}\left(a_{1} b_{21}+a_{2} b_{11}\right)\right] .
\end{aligned}
$$

It follows from (3.7) that $\varepsilon_{1}, \varepsilon_{2}, \delta_{1}, \delta_{2}$ are positive. After some algebra calculations, $\tilde{\mathbf{c}}$, $\hat{\mathbf{c}}$ satisfy (3.2), which ensures $\tilde{\mathbf{c}}$ and $\hat{\mathbf{c}}$ are coupled upper and lower solutions of the system (1.1). From (2.18), the quasisolutions $\overline{\mathbf{c}}$ and $\underline{\mathbf{c}}$ satisfy $\overline{\mathbf{c}} \geq \underline{\mathbf{c}}>0 .(2.17)$ is reduced to

$$
\left\{\begin{array}{l}
a_{1}-b_{11} \bar{c}_{1}-b_{12} \underline{c}_{2}-e_{1} \bar{c}_{1} \underline{c}_{2}=0=a_{1}-b_{11} \underline{c}_{1}-b_{12} \bar{c}_{2}-e_{1} \underline{c}_{1} \bar{c}_{2}, \\
a_{2}-b_{21} \underline{c}_{1}-b_{22} \bar{c}_{2}-e_{2} \underline{c}_{1} \bar{c}_{2}=0=a_{2}-b_{21} \bar{c}_{1}-b_{22} \underline{c}_{2}-e_{2} \bar{c}_{1} \underline{c}_{2} .
\end{array}\right.
$$

The relation (3.9) induces the following

$$
\left(b_{21} e_{1}-b_{11} e_{2}\right)\left(\bar{c}_{1}-\underline{c}_{1}\right)+\left(b_{12} e_{2}-b_{22} e_{1}\right)\left(\bar{c}_{2}-\underline{c}_{2}\right)=0 .
$$

Combining (3.7) and (3.10), we have $\bar{c}_{1}=\underline{c}_{1}=N_{1}^{*}, \bar{c}_{2}=\underline{c}_{2}=N_{2}^{*}$. By Theorem 2, we conclude $\lim _{t \rightarrow \infty}\left(u_{1}(x, t), u_{2}(x, t)\right)=\left(N_{1}^{*}, N_{2}^{*}\right)$.

\section{Discussions}

The main method in Section 2 is the upper and lower solutions and its monotone iteration. The technique is used in many papers for analysis of coupled parabolic systems, see, e.g. $[5,6,7,10,14]$. The virtue of the technique is by which the results of existence and stability is extended from the scalar equation to the coupled system. In particular, Pao and Ruan [20] have developed the method to deal with quasilinear parabolic system. Because iterative sequences in Section 2 are independent of the time, the delayed reaction terms of (1.1) can be obtained in analytical form. Our conclusions of Theorems 1, 2 are the extensions from the quasi-monotone nondecreasing reaction term of [20] to mixed quasi-monotone nondecreasing reaction term. By comparing the results between the quasilinear parabolic system and semilinear parabolic system [26], the conditions for the positive uniform equilibrium being asymptotic stable are coincident. It means that the theory of existence and stability in Theorems 1, 2 is usefulness and applicable to the general Lotka-Volterra type model. 
All of the previous phytoplankton models, such as $[2,3,9,11,12,23,24$, 25, 26], did not take density-dependent diffusion effects into account. In fact, plankton can move around subject to diffusion. Thus, it is more realistic to introduce the density-dependent diffusion of the plankton into the system. The concern of the density-dependent diffusion is reasonable in animal disperse model (see [22] for a review). The biological implications of Theorems 3 and 4 are that if the ratios of the intra-competition to the inter-competition belong to some parameter domain, the long term behaviours of the species tend to the positive uniform equilibrium. The two competitive species are coexistent. The results also have applicability to 3 species Lotka-Volterra model.

\section{References}

[1] D. Aikman and G. Hewitt. An experimental investigation of the rate and form of dispersal in grasshoppers. J. Appl. Ecol., 9:807-817, 1972.

http://dx.doi.org/10.2307/2401906.

[2] J. Chattopadhyay. Effects of toxic substances on a two-species competitive system. Ecol. Modl., 84:278-289, 1996.

http://dx.doi.org/10.1016/0304-3800(94)00134-0.

[3] F. Chen, Z. Li, X. Chen and J. Laitochova. Dynamic behaviors of a delay differential equation model of plankton allelopathy. Comput. Appl. Math., 206:733-754, 2007. http://dx.doi.org/10.1016/j.cam.2006.08.020.

[4] A. Friedman. Partial Differential Equations of Parabolic Type. Prentice Hall, Englewood Cliffs, NJ, 1964.

[5] S. Heikkila and V. Lakshmikantham. Monotone Iterative Techniques for Discontinuous Nonlinear Differential Equations. Marcel Dekker, Inc., New York, 1994.

[6] S. Koksal and V. Lakshmikantham. Unified approach to monotone iterative technique for semilinear parabolic problems. Dyn. Contin. Discrete Impuls. Syst. Ser. A Math. Anal., 10:539-548, 2003.

[7] G.S. Ladde, V. Lakshmikantham and A.S. Vatsala. Monotone Iterative Techniques for Nonlinear Differential Equations. Pitman, Boston, MA, New York, 1985.

[8] O.A. Ladyženskaja, V.A. Solonnikov and N.N. Ural'ceva. Linear and QuasiLinear Equations of Parabolic Type. Amer. Math. Soc. Transl., Providence, RI, 1968.

[9] Z. Liu and L. Chen. Positive periodic solution of a general discrete nonautonomous difference system of plankton allelopathy with delays. Comput. Appl. Math., 197:446-456, 2006. http://dx.doi.org/10.1016/j.cam.2005.09.023.

[10] R.H. Martin and H.L. Smith. Reaction-diffusion systems with time delay: monotonicity, invariance, comparison and convergence. J. Reine Angew. Math., 414:135, 1991.

[11] J. Maynard Smith. Models in Ecology. Cambridge University, Cambridge, 1974.

[12] A. Mukhopadhyay, J. Chattopadhyay and P.K. Tapaswi. A delay differential equations model of plankton allelopathy. Math. Biosci., 149:167-189, 1998. http://dx.doi.org/10.1016/S0025-5564(98)00005-4. 
[13] J.D. Murray. Mathematical Biology of Biomathematics Texts, vol. 19, 2nd edition. Springer-Verlag, Berlin, London, 2002.

[14] J.J. Nieto. An abstract monotone iterative technique. Nonlinear Anal., 28:19231933, 1997. http://dx.doi.org/10.1016/S0362-546X(97)89710-6.

[15] C.V. Pao. Nonlinear Parabolic And Ellipitic Equation. Plenum, New York, 1992.

[16] C.V. Pao. Dynamics of nonlinear parabolic systems with time delays. J. Math. Anal. Appl., 198:751-779, 1996. http://dx.doi.org/10.1006/jmaa.1996.0111.

[17] C.V. Pao. Systems of parabolic equations with continuous and discrete delays. J. Math. Anal. Appl., 205:157-185, 1997. http://dx.doi.org/10.1006/jmaa.1996.5177.

[18] C.V. Pao. Parabolic systems in unbounded domains. II. Equations with time delays. J. Math. Anal. Appl., 225:557-586, 1998. http://dx.doi.org/10.1006/jmaa.1998.6051.

[19] C.V. Pao. Quasilinear parabolic and elliptic equations with nonlinear boundary conditions. Nonlinear Anal., 66:639-662, 2007. http://dx.doi.org/10.1016/j.na.2005.12.007.

[20] C.V. Pao and W.H. Ruan. Positive solutions of quasilinear parabolic systems with nonlinear boundary conditions. J. Math. Anal. Appl., 333:472-499, 2007. http://dx.doi.org/10.1016/j.jmaa.2006.10.005.

[21] C.V. Pao and W.H. Ruan. Positive solutions of quasilinear parabolic systems with Dirichlet boundary condition. J. Differential Equations, 248:1175-1211, 2010. http://dx.doi.org/10.1016/j.jde.2009.12.011.

[22] N. Shigesada and K. Kawasaki. Biological Invasions: Theory and Practice. Oxford University Press, Oxford, 1997.

[23] C. Tian. Coexistence and asymptotic periodicity in a competition model of plankton allelopathy. Acta Appl. Math., 113:195-206, 2011. http://dx.doi.org/10.1007/s10440-010-9592-4.

[24] C. Tian and Z. Lin. Periodic solutions of reaction diffusion systems in a halfspace domain. Nonlinear Anal. Real World Appl., 9:811-821, 2008. http://dx.doi.org/10.1016/j.nonrwa.2007.01.001.

[25] C. Tian and Z. Lin. Asymptotic behavior of solutions of a periodic diffusion system of plankton allelopathy. Nonlinear Anal. Real World Appl., 11:15811588, 2010. http://dx.doi.org/10.1016/j.nonrwa.2009.03.012.

[26] C. Tian, L. Zhang and Z. Ling. The stability of a diffusion model of plankton allelopathy with spatio-temporal delays. Nonlinear Anal. Real World Appl., 10:2036-2046, 2009. http://dx.doi.org/10.1016/j.nonrwa.2008.03.016. 